\title{
The Global Risk Analysis as a tool to secure drug management of clinical trials in hospitals
}

\author{
Jacques A Weissenburger ${ }^{1,3}$, Juliette Godard ${ }^{1}$, Caroline Ly ${ }^{1}$, Anne Daguenel ${ }^{2}$, \\ Christine Fernandez $z^{2,3,4}$, Tabassome Simon ${ }^{1,3}$
}

${ }^{I}$ Department of Clinical Pharmacology \& Clinical research Platform (CRB, CRC, URC), Assistance-Publique Hopitaux de Paris, Paris, France, ${ }^{2}$ Depatment of Pharmacy, Assistance-Publique Hopitaux de Paris, PARIS, France, ${ }^{3}$ Sorbonne Universites, UPMC Paris Univ-06, PARIS, France, ${ }^{4}$ INSERM, Institut Pierre Louis, Epidemiologie et Sante Publique, France

Background: Errors during drug management of clinical trials are regularly reported to sponsors and regulatory agencies despite numerous standard operating processes. Risk management methods were developed to control the risks resulting from exposure of complex systems to a set of structural, functional, or cyclical hazards throughout their missions. We tested whether the Global Risk Analysis (GRA) method of risk management can be applied to secure the process of drug management (PDM) during clinical trials.

Material and methods: A multidisciplinary working group was formed to apply the 3-step GRA method to PDM. Step 1: The PDM was splitted into 24 consecutive functions. Interactions between these functions and predefined hazards were evaluated and those qualified as dangerous situations identified. Step 2: At least one scenario of accident was designed for each dangerous situation and their likelihood of occurrence (L) and severity (S) were assessed using specific scales. A decision matrix was defined to associate each (L, S) pair to one of the following acceptability criteria: C1: acceptable, C2: tolerable under control or C3: unacceptable. Step 3: Corrective actions were proposed according to C2 and C3 criteria and their feasibility assessed using predefined workload scales.

Results: Overall, 137 function-hazard interactions were recognized: 56 were identified as dangerous situations and 95 scenarios of accident were generated of which 53 were classified as C1, 42 as C2 and none as C3 acceptability. Drug dispending (33\%), prescription (18\%) and trial set-up (13\%) were the most vulnerable functions while human factor (56\%), information system (17\%) and work organization (8\%) were the most frequent sources of hazards. A $66 \%$ reduction of the number of $\mathrm{C} 2$ accident scenarios was obtained with 35 corrective actions including checklists of requirements prior to trial set-up; double-check procedures before drug dispending and administration of high-alert drugs and specific communication processes for different actors involved in the drug trial. Conclusion: GRA is a suitable tool to secure the process of drug management during in-hospital clinical trials. Its success depends on the quality of the multidisciplinary group commitment, its planning before the clinical trial set-up and the close follow-up of the corrective actions. 